\title{
Efficacy of floseal $\circledast$ vs tachosil@ vs no hemostatic agents for partial nephrectomy: A prospective multicenter comparative study (RECORd Project)
}

Eur Urol Suppl 2013;12;e887

Antonelli A. ${ }^{1}$, Minervini A. ${ }^{2}$, Arrighi N. ${ }^{3}$, Bianchi G. ${ }^{4}$, Cosciani Cunico S. ${ }^{3}$, Ficarra V. ${ }^{5}$, Fiori C. ${ }^{6}$, Martorana G. ${ }^{7}$, Lapini A. ${ }^{2}$, Medica M. ${ }^{8}$, Mirone V. ${ }^{9}$, Pinzi N. ${ }^{10}$, Porpiglia F. ${ }^{6}$, Rocco F. ${ }^{11}$, Rovereto B. ${ }^{12}$, Schiavina R. ${ }^{7}$, Serni S. ${ }^{2}$, Simeone C. $^{3}$, Terrone C. ${ }^{13}$, Volpe A. ${ }^{13}$, Zattoni F. ${ }^{5}$, Carini M. ${ }^{2}$, RECORd, More centres, Italy

${ }^{1}$ University of Brescia, Dept. of Urology, Brescia, Italy, ${ }^{2}$ University of Florence- Ospedale Careggi, Dept. of Urology, Florence, Italy, ${ }^{3}$ University of Brescia- Spedali Civili, Dept. of Urology, Brescia, Italy, ${ }^{4}$ University of Modena-Reggio Emilia, Dept. of Urology, Modena, Italy, ${ }^{5}$ University of Padua, Dept. of Urology, Padua, Italy, ${ }^{6}$ University of Turin- San Luigi Gonzaga Hospital, Dept. of Urology, Orbassano, Italy, ${ }^{7}$ University of Bologna, Dept. of Urology, Bologna, Italy, ${ }^{8}$ Civile Hospital, Dept. of Urology, Sestri Levante, Italy, ${ }^{9}$ University of Naples "Federico II", Dept. of Urology, Naples, Italy, ${ }^{10}$ Lucca Hospital, Dept. of Urology, Lucca, Italy, ${ }^{11}$ University of Milan, Dept. of Urology, Milan, Italy, ${ }^{12}$ Policlinico Hospital, Dept. of Urology, Pavia, Italy, ${ }^{13}$ University of Novara "L. Avogadro"- Azienda Ospedaliera Maggiore Della Carità, Dept. of Urology, Novara, Italy

INTRODUCTION \& OBJECTIVES: Aim is to evaluate the efficacy of FloSeal ${ }^{\circ}$ versus TachoSil囚 versus no hemostatic agents (HA) for partial nephrectomy (PN).

MATERIAL \& METHODS: Prospective observational multicenter study (RECORd Project) including the patients who underwent PN between January 2009 and January 2011 at 19 Italian centers. Clinical and surgical data were recorded in an on-line database and were managed by a single data-manager. The association between type of HA used and the need for blood transfusion/re-intervention and the risk of cardiovascular complications was evaluated.

RESULTS: 418 patients were recruited (231 had FloSeal 8 PN, 116 TachoSil 8 PN and 71 PN without any HA - pure PN). Among the 3 groups there were no differences in the rate of suture $(80.1 \%, 83.6 \%$ and $74.6 \%$, respectively) or coagulation of the tumor bed $(17.7 \%, 21.6 \%, 23.9 \%)$.

Postoperative bleeding requiring blood transfusion/reintervention took place in $9.3 \%, 7.9 \%$ and $15.7 \%$ of FloSeal $($, TachoSil $\Omega$ and pure PN, respectively (FloSeal $\Omega$ and TachoSil $\circledast$ vs pure $P N, p=0.05$ ), while cardiovascular complications in $0.9 \%, 0.9 \%$ and $5.7 \%$ (FloSeal $\circledast$ vs pure $P N, p=0.035$; TachoSil $\circledast$ vs pure $P N, p=0.05$ ). Multivariable analysis showed that preoperative haemoglobin level, lack of $\mathrm{HA}$ and $>50 \%$ endophytic tumor growth pattern were independent predictors of postoperative significant bleeding.

CONCLUSIONS: Both FloSeal $\circledast$ and TachoSil $\circledast$ seem to decrease the risk of significant postoperative bleeding. Only a few papers on the use of hemostatic agents (HA) during partial nephrectomy (PN) are reported. This study compares PN with Floseal $($, Tachosil $\circledast$ or without $H A$, showing that the use of $H A$ is protective against bleeding. 